\section{A SURVEY ON THE PREVALENCE OF HELICOBACTER PYLORI INFECTION PERFORMED IN A PEDIATRIC ENDOSCOPY UNIT DURING EIGHT YEARS}

\author{
V. Hurduc ${ }^{1}$, D. Plesca ${ }^{2}$, A. Zamfirescu², \\ M. Stefanescu², L. Bordei ${ }^{2}$ \\ ${ }^{1}$ Paediatric Gastroenterology, ${ }^{2}$ Paediatrics, \\ Victor Gomoiu Childrens' Hospital, Carol Davila \\ University of Medicine and Pharmacy, \\ Bucharest, Romania
}

Background and aims: Although Helicobacter pylori continues to be one of the most common bacterial infections worldwide, its incidence decreased recently in developed countries. The aim of this study is to assess the evolution of yearly prevalence of $\mathrm{H}$ pylori infection in symptomatic children evaluated endoscopically during 2001 2008.

Methods: We conducted a retrospective study on 688 consecutive symptomatic children (age range 6 months - 18 years; $F / M=418 / 270$ ) who required first upper endoscopy for various symptoms. The $\mathrm{H}$ pylori status was assessed by rapid urease test and histological examination. The prevalence changes of $\mathrm{H}$ pylori infection and their correlations with demographic characteristics and socioeconomic conditions were evaluated.

Results: H pylori infection was identified in 412 $(59,88 \%)$ of the 688 consecutive investigated children. Its yearly prevalence varied from $56,16 \%$ to $77,77 \%(p=0,242)$, with $70,29 \%$ in the last studied year. The following yearly values were observed: $56,16 \%, 48,38 \%, 46,25 \%, 46,42 \%, 49,01 \%, 77,77 \%$, $70,29 \%$. The prevalence of $\mathrm{H}$ pylori increased with age from $16,86 \%$ in the group 6 months - 3 years, to $36,48 \%$ for $15-18$ years. The colonization rate was inversely correlated with the socioeconomic status $(p<0,005)$.

Conclusions: The recent decline of $\mathrm{H}$ pylori infection observed in developed countries is not evident in our study, probably because the recent local economical changes did not result in sufficient decrease of its prevalence. The increase of the annual prevalence of $\mathrm{H}$ pylori infection in children can be probably justified by better diagnosis and by the selective character of the study.

\section{EPSTEIN-BARR VIRUS INFECTION AND GRAFT DYSFUNCTION IN THE FIRST YEAR POST-LIVER TRANSPLANTATION}

A. Dias ${ }^{1}$, D. Martins ${ }^{1}$, A.P. Campos ${ }^{1}$, C. Nogueira ${ }^{2}$, I. Gonçalves ${ }^{1}$

${ }^{1}$ Unidade de Hepatologia e Transplante Hepático Pediátrico, Hospital Pediátrico de Coimbra, ${ }^{2}$ Laboratório de Microbiologia, Faculdade de Medicina da Universidade de Coimbra, Coimbra, Portugal

Background and aim: EBV infection and posttransplant lymphoproliferative disease (PTLD) are closely linked, but the contribution of EBV to graft dysfunction (GD) has not been clearly reported. The aim was to evaluate the impact of EBV infection on GD post-liver transplantation (LT).

Methods: Retrospective study of children who underwent LT between 2004-2008. EBV status (serology and PCR) was determined before LT at 6 and 12 months $(\mathrm{m})$ or if GD was suspected. EBV positivity before LT was based on IgG and EBNAEBV (ELISA). Primary infection(PI)/reactivation were diagnosed by VCA-IgM or PCR-EBV+ (Real-time PCR). GD was accessed by cytolysis/cholestasis markers and/or histology.

Results: Forty children received a first LT and 32 were included. The mean age was 6 years $(5 \mathrm{~m}-15 \mathrm{y})$ and mean follow-up was 3y. Indications for LT were: biliary atresia(10), metabolic disease(8), alpha1antitrypsin deficiency(5), others(9). Before LT, 14 children, mean age 9.6y, were EBV-positive (group A) and 18 (56\%), mean age 3.2y, EBV-negative (group B). In group A there was no reactivation and in group $B, 12(67 \%)$ had PI. GD occurred in $71 \%$ of group $A$ vs $56 \%$ in group $B(p=0.471)$. EBV infection had no impact on the graft in 5/12 children. $7 / 12$ had another etiology for GD. Immunosuppression (ISS) was decreased in 6 (2 with suspected PTLD) with GD improvement in 1 and late rejection in 1.

Conclusions: EBV PI occurred in $67 \%$ of children after LT. Half experienced GD, but improvement was not associated with reduced ISS. Isolated EBV infection had a minor impact on GD. 